\title{
Ethnologies
}

\section{Patrimoine partagé et carnaval caribéen}

\section{Lucie Pradel}

Volume 34, numéro 1-2, 2012

URI : https://id.erudit.org/iderudit/1026153ar

DOI : https://doi.org/10.7202/1026153ar

Aller au sommaire du numéro

Éditeur(s)

Association Canadienne d'Ethnologie et de Folklore

ISSN

1481-5974 (imprimé)

1708-0401 (numérique)

Découvrir la revue

Citer cet article

Pradel, L. (2012). Patrimoine partagé et carnaval caribéen. Ethnologies, 34(1-2), 251-271. https://doi.org/10.7202/1026153ar

\section{Résumé de l'article}

Par sa représentation de diverses formes d'expressions patrimoniales, par son alliage de traditions orales, rituelles, sociales, de même que de savoirs et de savoir-faire, le carnaval est représentatif du patrimoine immatériel. Le terme " carnaval " minore la dimension d'un festival artistique animé par la musique, la danse, le théâtre, l'art de la performance, la peinture, la sculpture, le stylisme, la chapellerie, l'art photographique. Le carnaval se singularise par son rôle d'incubateur de nouvelles pratiques et par son influence dynamique sur d'autres secteurs d'activités, à savoir les secteurs artistique, culturel, social et économique. La notion de patrimoine partagé est utile en tant que mode d'interprétation du carnaval et, de manière plus large, cette notion contribue également à l'interprétation du patrimoine immatériel.
Ce document est protégé par la loi sur le droit d'auteur. L'utilisation des services d'Érudit (y compris la reproduction) est assujettie à sa politique d'utilisation que vous pouvez consulter en ligne.

https://apropos.erudit.org/fr/usagers/politique-dutilisation/ 


\section{Patrimoine partagé et CARnaVAl CARIbÉEN}

\section{Lucie Pradel \\ Université des Antilles et de la Guyane}

Le patrimoine culturel immatériel, en tant que nouvelle catégorie patrimoniale, ne manque pas de susciter l'intérêt mais soulève des interrogations sur sa place et sur son rôle auprès des individus et au sein de la société. Ces questionnements impulsent une démarche de retour sur le terrain, utile à la mise en adéquation avec le débat engendré autour de ce patrimoine mais également indispensable à une meilleure connaissance et interprétation de cet héritage porteur de valeurs auxquelles les individus et les populations sont attachés. Par ses combinaisons de traditions orales, rituelles, sociales, de même que de savoirs et de savoir-faire, le carnaval est représentatif du patrimoine immatériel. De Binche à Barranquilla ou à Oruro, il est hissé au plus haut rang. Dans la Caraibe, l'alliage entre pratiques et traditions de l'Ancien et du Nouveau Monde lui confère son caractère essentiel; en réalité, le terme « carnaval » minore la dimension d'un festival artistique animé par la musique, la danse, le théâtre, l'art de la performance, la peinture, la sculpture, le stylisme, la chapellerie, l'art photographique. En tant que conservatoire, il consigne des pratiques et des traditions ayant pour finalité d'être partagées selon des modalités renouvelées par notre contexte postmoderne.

La notion de patrimoine partagé se situe dans la lignée de celle de patrimoine métissé dont l'accent souligne les mélanges, les contacts, les interactions, les échanges entre individus et entre groupes (Turgeon 2003). Dans les sociétés créoles dont fait partie notre terrain, la Guadeloupe, le patrimoine métissé permet le partage de traditions et de pratiques entre des populations historiquement très diversifiées. Le carnaval se singularise par son rôle d'embrayeur ; il confère la priorité à la créativité, à l'initiative personnelle et collective. En tant qu'incubateur de nouvelles pratiques, le carnaval dynamise d'autres secteurs d'activités, comme les secteurs artistique, culturel, social et économique. L'intégration de ces nouvelles pratiques concourt à l'intégration de ses pratiquants et à leur participation 
à la vie de la collectivité. La notion de patrimoine partagé se prête à l'interprétation du carnaval et de manière plus large à celle du patrimoine immatériel.

\section{Un temps fort de la vie culturelle}

Le carnaval, en tant que grande fête populaire, constitue un temps fort de la vie culturelle ; il rassemble des milliers de participants et de spectateurs dans la manifestation festive la plus longue de l'année. Les festivités débutent le premier dimanche du mois de janvier, avec en prémisses, le bain de la Saint-Sylvestre, ou ben démaré1. Le carnaval atteint son apogée pendant six jours, du vendredi précédant le Mardi gras au mercredi des Cendres. La fête laisse place au début du carême, mais est réitérée de façon éphémère pendant une journée, « le jeudi de la mi-carême ».

À partir du mois de janvier jusqu'aux jours gras, les défilés se tiennent chaque dimanche dans différentes villes ou communes selon un calendrier élaboré chaque année par les comités du carnaval. En marge ont lieu des manifestations communes : animations musicales, expositions, élection de la reine du carnaval, bals, ou des manifestations spécifiques proposées par un groupe carnavalesque. En 2011, la Guadeloupe comptait 130 groupes actifs et environ 20000 membres (Raboteur et Landais-Raboteur, s.d.), chiffres relativement importants pour une population de 404635 habitants (Recensement de 2011).

La popularité de cette fête influe sur notre approche ethnologique de terrain, puisque dès le début du mois de janvier, la couverture médiatique du carnaval est lancée avec des reportages télévisés et radiophoniques, des interviews, des débats hebdomadaires qui s'intensifient pour devenir quasi quotidiens pendant les jours gras. Le carnaval en tant qu'événement social est propice au regroupement familial : l'on y vient dès le plus jeune âge et en famille, l'on y grandit, en tenant tour à tour ou uniquement le rôle de spectateur et/ou de participant. La gratuité de l'événement facilite sa pérennité et l'assiduité des spectateurs pendant un trimestre. Outre cette immersion et l'adhésion depuis plusieurs années à des groupes carnavalesques, notre approche ethnologique a pris la forme d'entretiens, utilisés par la suite dans des travaux de recherche, auprès de deux groupes ayant contribué au renouveau du carnaval et qui ont créé un mouvement culturel dans les deux villes principales : Akiyo, à la fin des années 1970 à

1. Ben démaré : le bain purificateur afin de bien démarrer l'année est composé avec des plantes médicinales : voir, en ligne, Rimèd razyé : féyaj pou premyé dlan! (Les références des sites Internet mentionnés dans le texte se trouvent en fin d'article.) 
Pointe-à-Pitre, et Voukoum, au début de 1990 à Basse-Terre. Ces entretiens se sont déroulés avec 24 membres du groupe Akiyo (dont les principaux fondateurs) sur une durée de deux ans entre 2007 et 2009, période pendant laquelle nous avons pris part aux activités du groupe. Parallèlement, des entretiens se sont tenus avec le président du groupe Voukoum en 2007 et de nouveau en 2014 alors qu'il a cédé son siège de président mais demeure fortement impliqué et actif au sein du groupe en qualité de vice-président.

\section{Traits caractéristiques du carnaval de la Guadeloupe}

La diversité du carnaval reflète celle des groupes dont le nombre moyen de participants varie entre cinquante et cent personnes mais qui peut atteindre plus de mille adhérents pour le plus important en nombre. Les critères d'adhésion aux groupes les plus anciennement implantés révèlent le caractère familial de la manifestation à l'origine, avec des participants unis par des liens biologiques ou adhérant à des valeurs partagées². Le groupe peut être représentatif d'un quartier, (Raizet City, années 1980), ou partiellement d'une profession (les cuisinières parmi les membres du Point d'Interrogation, années 1990) ; une tradition musicale (les derniers membres des Mas à Senjan parmi les membres du groupe Akiyo, fin des années 1970). La tradition de musique live et de danse, en renforçant la contiguité entre musique, danse et carnaval, attire un grand nombre de musiciens et de danseurs vers le carnaval. Pareillement, la proximité de cette célébration avec la période de Noël qui draine un grand nombre de participants vers les chanté nwel, (cantiques et chants de Noël avec accompagnement musical) favorise un rapprochement entre ces différents adeptes de la fête (voir, en ligne, Kasika, « Nwel a fon kako », et Cactus Cho, «Chantons les louanges »).

La diversité des styles (musiques, chants, choix des matériaux vestimentaires) jusqu'aux parcours empruntés par les groupes (centreville, périphérie urbaine) constitue un autre trait distinctif des groupes. L'affirmation d'une identité culturelle a donné naissance à Akiyo à la fin des années 1970, à l'expression d'un mouvement culturel et artistique et au maintien de traditions. La propension pour les « mas » (terme générique pour désigner la manifestation et ses participants, utilisé par les « group a po » pour se distinguer du carnaval) a largement débordé le cadre de l'agglomération de Pointe-à-Pitre où le groupe a pris naissance pour s'étendre au reste de l'archipel et de la nation (pour l'exemple des

2. Telle est par exemple l'origine du groupe Magma, groupe emblématique du Carnaval implanté dans la ville de Basse-Terre (voir le site du groupe en ligne). 
groupes franciliens, voir en ligne : «Bwa Bandé », Carnaval tropical de 2013 ; "Choukaj Mas Paris » ; " Miyo et Maskadom » à Longjumeau ; «Mas Mélé »). L'usage de tambours recouverts de peaux de cabri a défini leur appellation de « group a po ».

Des projets en faveur des jeunes, dont certains à caractère social, sont à l'origine d'Atafaya (2001) et de Mas Moul Massif (2002), groupes composés majoritairement d'adolescents, concept nouveau dans le paysage carnavalesque traditionnellement tourné vers l'intergénérationnel, mais créateur d'émulation et adopté par un nombre grandissant de groupes et de jeunes qui y trouvent une manière appropriée de s'exprimer et d'être représentés (voir, en ligne, « Atafaya, » Sainte-Anne, Abymes 2013 ; « Mas Moul Massif »). Ce désir de représentation anime différents groupes, comme Amazone, composé uniquement de femmes, ou Kiss (Karukera International Stars System) partagé par les gays, lesbiennes et hétéros. Le pays ou la région d'origine peut devenir critère de représentation : Haïti est représentée par le groupe Tambou Guinen, Assoto Guinen ou Family Band ; le Brésil par Batala Gwada ; la Bretagne par le groupe Bagag Karukéra. Néanmoins, l'adhésion à un groupe n'ayant pas de caractère obligatoire, certains choisissent de former des duos, des trios ; le nombre peut encore être en augmentation ou en diminution car le carnaval est une scène ouverte aux performeurs. La prestation solo, pourvu qu'elle ait un caractère artistique ou original, ravira également le public.

\section{Couleur musicale}

La musique opère également la distinction entre les groupes et guide le choix des nouveaux adhérents, particulièrement ceux désireux d'intégrer le chœur des musiciens. Les « group a po » représentent ceux dont la section musicale prend le plus d'ampleur ; cet attrait favorise la transmission informelle de la musique et la prise en charge des nouveaux musiciens par les anciens. Les fréquences du grave à l'aigu ont donné lieu à la création par Akiyo de trois types de tambours : basse, contrebasse, tanbour-chant ; ce dernier est un tambour d'aisselle, percuté à l'aide de baguettes. La section rythmique accueille également des percussions cucurbitales (calebasses) et des strombophones (conques de lambi). Le fouet manié par les enfants et adolescents en début de cortège entre dans l'alliage musical des airs enregistrés (voir, en ligne, "Akiyo »; « Nasyion a neg mawon »; " Mas ka kle»).

Les groupes avec des caisses claires rassemblent des musiciens confirmés, 
trompettistes, saxophonistes. Aux côtés des caisses claires se retrouvent les tambours en matière plastique, objets de récupération ayant servi au transport de denrées alimentaires. Après nettoyage et décoration aux couleurs du groupe ou du thème choisi, ils acquièrent une nouvelle assignation. Ces musiciens recourent comme les précédents aux conques et aux calebasses (voir, en ligne, «Stars \& Tany's Girls »).

Les groupes avec des synthétiseurs, plus implantés dans la partie sudouest de l'île et la ville de Basse-Terre, privilégient les instruments électroanalogiques. L'orchestre inclut les synthétiseurs, les guitares électriques, mais également les baffles, les groupes électrogènes et les micros mis à disposition des chanteurs. Le reste de la section musicale des groupes à synthés égale celle des groupes à caisses claires dont ils se distinguent néanmoins par leur tempo plus rapide et énergique (voir, en ligne, « Concours Synthé.mpg »; «Pikan final »; «TV97.Net »; «Char pikan »).

Les groupes avec des steel pan ont renoué avec la tradition du steel qui se pratiquait dans quelques communes de l'île. L'inclination pour cet instrument a fait naître un nouvel engouement et les formations musicales accueillent des instrumentistes de plus en plus jeunes (voir, en ligne, «Karukéra Stee »).

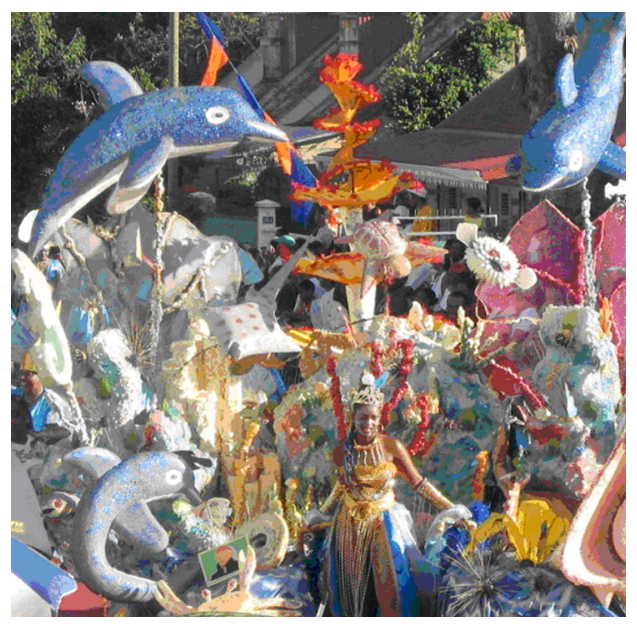

Figure 1. «Mer-veilleuse », groupe avec des caisses claires.

Ces critères de distinction s'estompent dès lors que l'on tient compte du rôle d'unité du tambour présent sous toutes ses formes dans le carnaval. Il s'avère indispensable au groupe «Ka sanblé » qui, dans sa version originale, en a fait son instrument phare. Les groupes à synthés ne l'ont pas délaissé ; il contribue au renforcement rythmique dans les groupes à caisses claires. Repensé et réinventé, il s'accorde au besoins des "group a po »; adopté par « Bagag Karukéra », le ka figure aux cotés du biniou et de la bombarde; il s'harmonise à la samba-reggae bahianaise de « Batala Gwada », et s'intègre depuis jadis aux vaccines haïtiennes. 


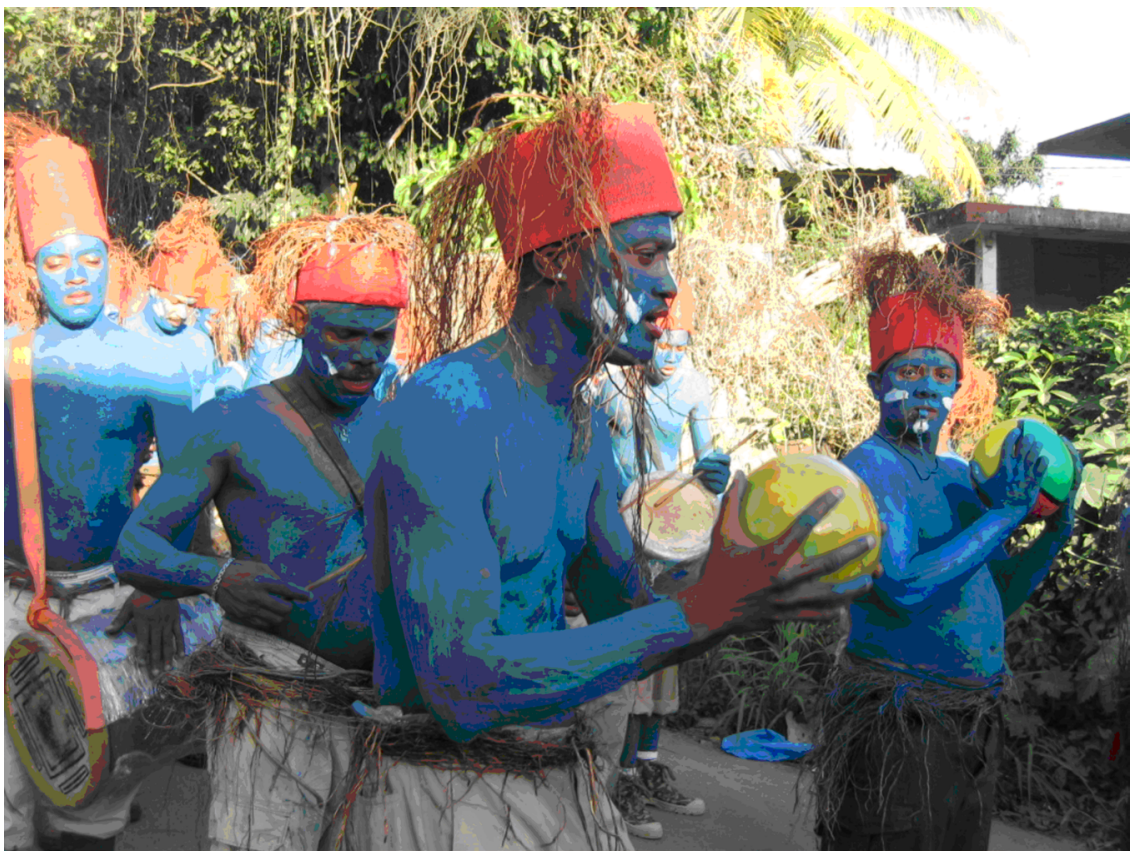

Figure 2. «Voukoum », Group a po, 2007.

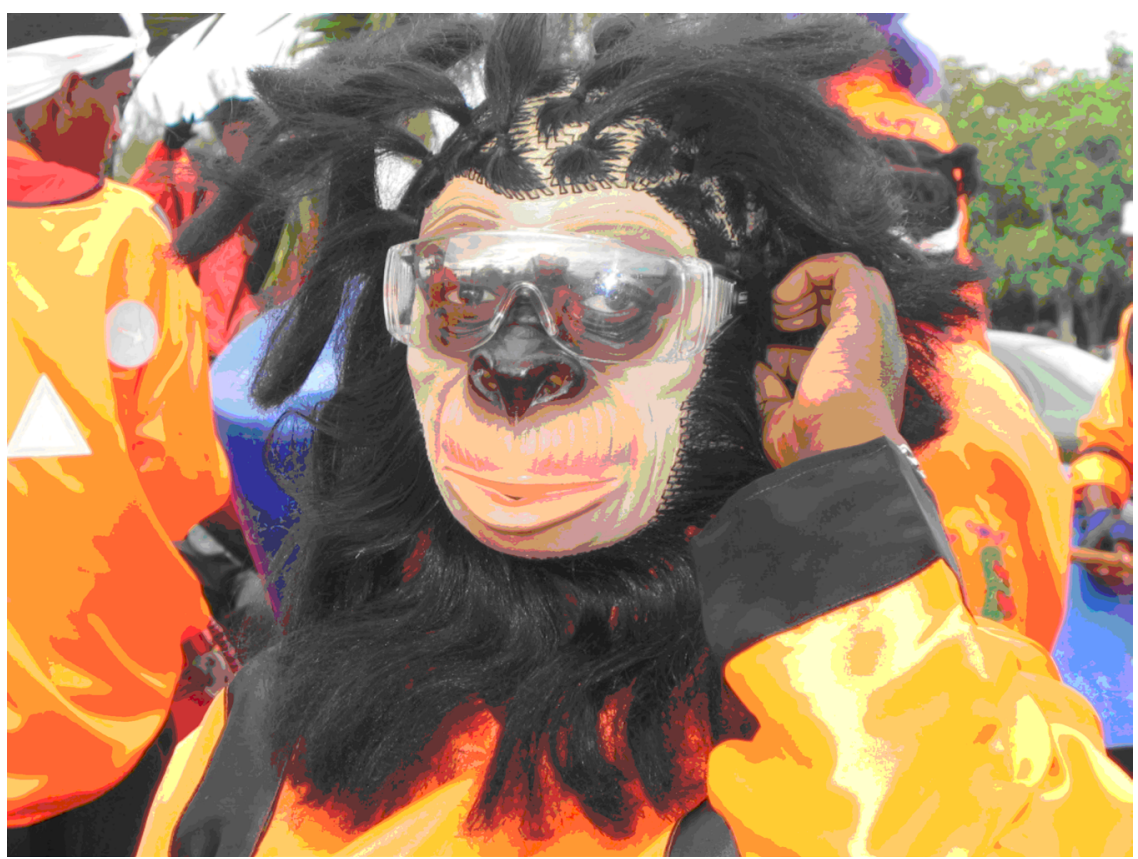

Figure 3. Membre d'un groupe avec steel pan. 


\section{Rôle d'incubation de pratiques}

La majorité des groupes sont constitués en structure de type associatif Loi 1901 et de ce fait, la participation aux défilés carnavalesques ou aux « déboulés », pour reprendre une terminologie propre aux " group a po », suggérant une marche plus rapide mais cadencée, n'est qu'une partie des nombreuses activités inscrites à un calendrier qui se décline sur une base annuelle, mensuelle, hebdomadaire. Généralement, les calendriers incluent les activités musicales, chorégraphiques mais aussi sportives et récréatives qui se traduisent par des tournois sportifs, des sorties découverte de la nature, des séjours touristiques hors de l'île.

Le carnaval en tant que lieu de transmission permet par la tenue d'ateliers de s'initier au style musical du groupe d'adhésion et dans le cas des « group a po » de s'initier à la confection des instruments de musique. Cette transmission informelle se déroule en deux temps : pendant la période précédant les jours gras où l'on peut obtenir des conseils et de l'aide pour la confection de nouvelles tenues ou pour le perfectionnement de la pratique instrumentale ; pendant le reste de l'année où le calendrier mensuel ou même hebdomadaire pour certains groupes permet de prendre part aux activités sociales et de loisirs proposées aux adhérents.

La transmission de la pratique musicale a contribué à l'évolution des écoles de musique à travers l'engouement suscité par les groupes en vogue. Pour donner un exemple récent, l'avènement des groupes à steel pan et la popularité d'un de ses groupes phares, «MMM » (Mas Moul Massif), a contribué à propager auprès des jeunes l'art d'un instrument que ses détenteurs âgés voyaient péricliter. Les concerts, la production de vidéos, de Cds, dont un single avec Admiral T, artiste célèbre de la scène dancehall, ont contribué à l'élargissement de l'offre de cours des écoles de musique qui, à leur programme, ont ajouté les cours de steel pan. Par ailleurs, cette collaboration entre groupe carnavalesque et artiste dancehall vient enrichir de manière inédite le répertoire musical local (voir, en ligne, « Arété sa »). L'avènement des groupes à steel pan est à mettre en parallèle avec celui des « group a po » qui à leur époque ont dynamisé le carnaval et enrichi la culture par la création de nouveaux instruments, d'un nouveau style musical, par la production de musique enregistrée, de concerts agrémentés de nouvelles chorégraphies et scénographies ; ceci définissant un autre rapport au tambour qui depuis longtemps demeurait déprécié et marginalisé. De ces deux expériences sont nés des styles musicaux viables qui, au-delà de la saison carnavalesque, animent la vie culturelle et ouvrent de nouvelles frontières. 
Le carnaval étant une fête éphémère, elle donne lieu chaque année à de nouvelles créations : vestimentaires, musicales, chorégraphiques, artisanales ; le stylisme y trouve une place grandissante même si, dans le passé, les déguisements n'impliquaient aucun investissement financier conception à laquelle continuent d'adhérer les " group a po ». Il suffisait simplement d'enfiler par dérision quelque vêtement usagé qui bien souvent provoquait le rire. La compétition à laquelle donne lieu aujourd'hui le carnaval, par sa médiatisation, son potentiel et ses retombées artistiques, touristiques et économiques, avive parmi les groupes, particulièrement les groupes à caisses claires et les groupes à synthétiseurs, le désir de remporter les suffrages du public, de répondre aux exigences de la photographie, d'acquérir une notoriété et, pour y parvenir, de faire appel aux professionnels de la mode. Même si la «brésilianisation » des tenues est un phénomène récent et ne s'accompagne pas des chars allégoriques sud-américains, elle est suffisamment encouragée par les prix décernés lors des concours pour se propager rapidement. Bien que les ateliers d'aide à la confection des tenues existent au sein des associations, la sophistication des tenues pousse au professionnalisme et à la collaboration entre carnavaliers et stylistes ; la saison carnavalesque est quelquefois couplée à des défilés de mode. En fait, le secteur du stylisme n'est pas seul concerné mais aussi celui de l'esthétique, de la remise en forme, voire de la psychologie par thérapie de groupe. Les salles de sports bénéficient d'une affluence spéciale avant la saison des fêtes ; elle est moindre pendant le carnaval pour ceux qui considèrent ce lieu de rendez-vous festivalier annuel comme la plus grande salle de remise en forme. Ainsi, par le renforcement du rapport entre domaine festif, culturel et économique, le carnaval suscite de nouvelles pratiques. Les fêtes célébrées pendant les jours gras mettent en valeur le patrimoine culinaire, en invitant une dernière fois à faire bonne chère avant le carême, synonyme pour certains sinon de jeûne, du moins de frugalité.

La célébration de ce festival populaire, en faisant appel au bénévolat, n'exclut pas pour autant les professionnels mais les intègre au rythme de l'évolution de cette expression patrimoniale; ceci implique néanmoins des choix et des équilibrages. Si la présence artisanale tend à reculer du fait du repli de la production de décors, pour l'édition de 2014, les plasticiens ont été invités, par le biais d'un concours, à produire de nouveaux masques, afin de rompre avec la monotonie des déguisements de facture industrielle. Cet appel à plus de rigueur ne plaide pas uniquement en faveur des professionnels car la distance entre le carnaval et le spectacle étant très courte, de nouvelles perspectives s'ouvrent aux participants-amateurs en dehors de la 
période des festivités. Certains groupes en vue sont régulièrement invités à effectuer des prestations musicales et chorégraphiques à l'intention des touristes de passage ou, à l'occasion, de participations à d'autres carnavals (Brooklyn, Nice, Notting Hill, etc.). La prestation de ces artistes-amateurs dirige les pas vers l'émulation, vers un certain professionnalisme et vers de nouveaux produits d'appel touristique à l'intention d'une clientèle étrangère potentielle.

\section{Pratique festive et pratique solidaire}

Dans le passé, les regroupements par le biais desquels les membres cotisaient afin d'enterrer les morts et venir en aide aux vivants étaient désignés sous le nom de "sociétés » qui, par la suite, donneront naissance aux mutuelles (voir, en ligne, l'historique de la mutuelle Mare Gaillard). Un rapport d'administration au début de la Révolution française montre ces "sociétés » dont les membres forment des "nations », des groupes ethnolinguistiques ayant pour activités « des danses, réunions, collations, convois funèbres ». Tout comme la pratique festive étaye la pratique solidaire, la pratique des « mas » se veut en lien avec l'individu au sein de sa société. Certains groupes poursuivent ce but à travers des projets d'insertion sociale. Pour Voukoum, qui intervient depuis une vingtaine d'années sur le territoire local et national hexagonal dans des opérations d'intégration de jeunes issus de quartiers dits difficiles, l'action d'accompagnement de l'individu est afférente à la pratique des « mas »; elle vise actuellement à prendre la forme d'un projet de création d'un centre culturel pédagogique professionnel à l'intention de jeunes en chantier-école. Dans ce cadre, le jeune hébergé devra mobiliser une part de temps pour des projets culturels. D'autres actions à impact social ont pris la forme d'ateliers de fabrication de tambours, d'ateliers de musique gwosiwo (dénomination du style musical de Voukoum), d'actions en direction des personnes âgées et des handicapés. À leur intention, des itinéraires ciblés sont organisés par les « mas » afin de permettre l'inclusion du plus grand nombre à la fête. Dans un cadre pédagogique proposé aux plus jeunes dont les parents prennent part aux ateliers de Voukoum, des aides au travail scolaire sont mises en place (remise à niveau, aide au devoirs); pour tous les publics, une méthode orale d'apprentissage de la percussion, basée sur des onomatopées, a été créée.

Le partage avec les jeunes découle de leur forte présence au sein de l'association et dans la ville où se situe le local, à la périphérie de BasseTerre au Bas-du-Bourg. Il relève également de l'adhésion de Voukoum à un réseau international tourné vers les jeunes des favelas de Sao Paulo, 
des townships de Johannesburg ou vers les déshérités de Roumanie. Lors d'invitations à des festivals, Voukoum propose d'animer des ateliers afin exposer sa démarche et sa philosophie. Celle-ci s'enracine dans le fait que le « mas » est une transformation de l'être et que l'engagement culturel porte l'individu au respect de soi et de l'autre, au respect du bien d'autrui et des règles fondamentales du vivre ensemble. De ce fait, le lieu d'évolution des « mas » est un lieu de vie, un lieu de consensus, un axe structurant : un enfant peut rappeler un adulte à l'ordre car la cohésion entre les membres vient du rapport égalitaire entretenu par tous. Les tenues portées lors des déboulés par leur dépouillement ou leur uniformité suggèrent l'égalité. En enlevant le vêtement social, en endossant la tenue Mas a tè et feyaj gwadloup (argile et feuilles), le corps retrouve la liberté. L'argile et l'élément végétal renvoient à l'environnement local et à la place de l'individu dans la nature. De façon générale, les tenues des « mas » procèdent d'une triade : nature, histoire, patrimoine. Dans cette zone de confluences, le « mas » interroge, interpelle et partage. Chaque année, les thèmes sont abordés à l'aune de dimensions historiques, culturelles, environnementales, sociétales et sociales. Outre le fait qu'il soit un lieu d'activités communes (patrimoniales, artistiques, sportives, activités de pleine nature et de découverte), la finalité de Voukoum réside dans l'aide apportée à l'individu afin de l'aider à se fortifier, à faire face à la vie et ses aléas. Le médium choisi est celui de la connaissance de soi, de son environnement, de sa culture, de son histoire. Beaucoup de membres ont pris naissance parmi les « mas », y ont grandi, et à l'âge adulte, ceux qui quittent le département parfois pour poursuivre des études se voient remettre des supports artistiques visant à les aider symboliquement à surmonter les moments difficiles sans céder à la déprime ou à la dépression. À Bas-du-Bourg, la prise en charge à la fois symbolique et réelle des plus fragiles par le groupe vise à limiter leurs risques d'isolement, d'exclusion, les risques de dérive pouvant les mener à la privation de liberté.

\section{Carnaval caribéen}

Aucun chiffre précis ne permet de dénombrer la diffusion des carnavals caribéens dans le monde, sinon de manière catégorielle : plus d'une soixantaine de festivités carnavalesques caribéennes se tiennent tous les ans dans des villes d'Amérique et d'Europe. Elles représentent les fêtes transnationales les plus populaires du monde (Manning 1990: 6). Par la création d'interstices géoéconomiques et géopolitiques, elles concourent au renforcement du tissu économique (Nurse 2004) ${ }^{3}$. Dans la Caraïbe, l'histoire

3. Par exemple, le festival de Notting Hill à Londres attire plus deux millions de 
du carnaval révèle celle des populations ; elle témoigne des changements, des transformations sociales, économiques et politiques de même que de leurs impacts sur les individus et sur la société. Les dynamiques, tout comme les conflits de race, de genre, de classe sont hissés sur cette plate-forme carnavalesque.

La définition du carnaval peut résulter d'un double regard et d'une double interprétation : «It is a tool of political patronage, cynical racial manipulation and commercial opportunism. To others, the Carnival remains the medium through which hegemony may be countered, wherein racial, gender, and class stereotypes, stigmas and hierarchies may be challenged, questioned, and subverted "(Green et Scher 2007 : 7). Les écrits sur le carnaval de la métaspora font écho à la portée politique de cette célébration, de ses implications sur les communautés, de son influence sur la construction, l'affirmation et le maintien d'une identité. En tant que medium artistique multi-expressif, le carnaval a trait à l'identité. À Londres, à Leeds, il institue de nouvelles subjectivités, « de nouveaux concepts de l'identité noire britannique, dans laquelle les arts expressifs et autochtones constituent une caractéristique centrale » (Connor et Farrar 2004 : 256, traduction libre). Il informe des changements ou des continuités dans une des sphères d'observation de l'évolution des communautés antillaises de New York ; le Brooklyn's Labor Day Carnival fait état d'un double potentiel, « des politiques à la fois conformistes et d'opposition y étant toujours présentes simultanément » (256, traduction libre), par le nivellement des différences et l'unité de circonstance provoqués par cette célébration. Les carnavals caribéens couvrent différents espaces géographiques retraçant des déplacements de populations. Stables et éphémères à la fois, ils concourent à définir des communautés ou à les reconfigurer.

La chronologie carnavalesque, en tant que témoignage sur la société et sur ses membres, s'établit à partir de faits marquants. L'émergence des « group a po » en Guadeloupe a coïncidé avec un moment de lutte pour l'autodétermination et pour l'affirmation d'une identité culturelle; celle des groupes à steel pan est plutôt représentative du contexte postmoderne. En Jamaïque, le carnaval qui débuta avec le Roots Jonkonnu au sein duquel on comptait Cowhead, Pitchy Patchy, Devil, Warrior, Indian, s'accrut par l'addition de nouveaux personnages issus des «Fancy Dress »- le roi, la reine, la jeune fille (Flower Girl) - ou représentatifs de la société

personnes en deux jours et impacte l'économie de plus de 93 millions de livres ; le Labor Day à New York rassemble 3,5 millions de personnes, et le Caribana à Toronto un million. 
en évolution - le Capitaine, l'agent de police, la femme enceinte... Les «Fancy bands » étaient identifiables par leur langage supra-verbal qui, audelà de Trinidad, a trait au statut social, mais le costume dénote également les influences subtiles qui ont marqué le carnaval, comme l'orientalisme insinué par les commerçants syro-libanais à travers leur choix de couleurs et d'étoffes proposées dans leur magasins pour la confection des costumes. L'iconographie guadeloupéenne rapporte parmi les critères esthétiques des Indo-descendants lors de la fête de Pongal, des costumes élaborés ornés de petits miroirs qui siéent aux contextes festivaliers que l'on retrouve dans les «mas» de Vieux-Fort ${ }^{4}$. Néanmoins, perpétuer la tradition ne signifie pas rester résolument tourné vers le passé et les groupes ne manquent pas à travers leurs choix de thématiques d'interpeller sur des sujets contemporains. La tenue " code-barre » portée par les membres du groupe Akiyo est un vêtement blanc sur lequel est imprimée la série parallèle d'un code barre unidimensionnel : une façon de rappeler symboliquement l'uniformisation et les inversions mentales induites par la numérisation.

\section{Du mythe au carnaval}

L'historiographie rétrospective par le retour du rite adossé au mythe apporte, outre des indices de compréhension du carnaval, une clarification de sa symbolique et un complément à la définition du patrimoine partagé. Dans la Caraïbe, la géomorphologie de l'île a influencé la composition des cultes et la pratique des rituels. Les figures mythiques, en investissant ce lieu, ont réitéré le rapport constant entre l'homme et la nature par le rite et par le mythe. Les êtres surmoïques ont apposé leurs empreintes dans des domaines représentatifs - forestier et surtout aquatique. L'eau sous toutes ses formes s'associe aux déités de rang majeur : d'une calebasse cosmique amérindienne surgit la mer ; en Haïti, le couple primordial Dambalah et Aida Oueddo ont pour principe l'eau; à Cuba, la reine de güemilere (la fête), Ochun, a pour lieux de prédilection les rivières, les fontaines, les eaux fraîches. Elle quitte son palais aquatique aux premiers sons des tambours pour se rendre à la fête où l'attendent les humains qu'elle gratifie de sa joie, signe de félicité et de prospérité. Son amant Chango, dont la sphère d'influence s'étend à la fois aux Antilles et aux continents africain et américain, est roi du güemilere et passionné de tambour. La forêt alloue à la fête le tambour, ce médium privilégié ; son « bois fouillé », tronc évidé résultant de la coupe de l'arbre, figure dans les mythologies la jonction entre la terre et le ciel.

Les populations originaires d'Afrique transférées dans le Nouveau

$4 \quad$ Ils font partie des plus anciens groupes dans la tradition des masques. 
Monde emportaient dans leur mémoire des supports essentiels : le souvenir des dieux, des mythes, des rites, des rythmes, des contes, des légendes, des proverbes, des chants, des danses, des sculptures, vecteurs fondamentaux de leur pensée religieuse et qui trouvèrent dans des repères profanes, dont celui carnavalesque, un lieu de survivance (Pradel 2000). En Haïti, « la musique de carnaval a emprunté au vaudou son phrasé, ses échelles heptatoniques et pentatoniques, des gammes ecclésiastiques, tout comme ses rythmes les plus vifs combinés à d'autres éléments de la musique populaire » (Voegeli 1994 : 234). En Guadeloupe, bien que la pudeur lui ait imposé aujourd'hui de dissimuler son anatomie, Vaval, roi phallique du carnaval, a toujours mené le cortège de ses adeptes en exhibant fièrement son organe viril. Sa présence en tête de cortège est signe de réjouissance et de gaité. Les rois, les costumes, les masques, la convivialité, la liberté de geste et d'expression, voire la satire, apportent à cette manifestation festive son cachet distinctif.

\section{Conservatoire de pratiques et de traits communs}

L'approche historiographique éclaire les étapes de patrimonialisation mais également l'inscription d'une mémoire artisanale dans le carnaval (voir par exemple, en ligne, l'utilisation de la fibre de coco et l'hommage à saint Georges chevalier par les artisans, ainsi que l'art floral au Carnaval de Guadeloupe en 2007). Les principales cultures s'y trouvent représentées et en premier lieu la monoculture cannière, symbolisée par les cornes de l'animal de charrue qui, de manière représentative à travers le monde, symbolise la lune cornue, le temps de fécondité. Un produit dérivé de la transformation industrielle de la canne, le gwosiwo (mélasse) entre dans la composition du « mas gwosiwo » et du « mas a Congo ». La corde de chanvre servant à confectionner les fouets, quand elle est dénouée, stylisée ou agrémentée d'accessoires, devient un élément vestimentaire pour les « mas » des « group a po ».

\section{Mas, initiation et végétation}

L'activité bananière a donné naissance à une tenue végétale composée de feuilles sèches du bananier. Les feuillages contribuent à la composition de différentes tenues ${ }^{5}$. Jack-in-the-Green, membre emblématique du Jonkonnu jamaïcain, endosse un costume fait entièrement d'éléments végétaux. Cette utilisation des feuilles, selon Judith Bettelheim, existe dans diverses régions de la zone subsaharienne, et de façon notoire chez les Mandingues 5. Judith Bettelheim (1976) associe cette tenue des carnavaliers au vêtement africain porté lors de l'initiation. 
de Gambie. Le fara-kankurang mandingue revêt un costume végétal lors des cérémonies de circoncision (Bettelheim 1976:21-27). Toujours en lien avec les activités artisanales, le coton et les grains de café entrent dans la panoplie festivalière en faisant partie de la décoration des costumes, ou en donnant leur thématique pour une illustration en décors. À l'instar du tonneau utilisé pour le transport de denrées alimentaires puis par la suite reconverti à des fins instrumentales, la fibre textile extraite du jute ayant dans le passé servi au conditionnement des produits d'exportation fait partie des étoffes priorisées par les « group a po ».

\section{Jabs/devils}

Les figures traditionnelles antillaises partagent des traits hérités à la fois de l'immigration et des déplacements intra-caribéens. En 1794, la victoire de Victor Hughes en Guadeloupe et la menace de la guillotine avaient précipité le départ des colons vers les îles anglaises et vers Trinidad. En 1873, la promulgation de la Cédula de población favorisa l'arrivée à Trinidad d'immigrants catholiques de la Louisiane, la Martinique, Grenade, SainteLucie, la Dominique, Saint-Vincent, Nevis, Haïti et la Guadeloupe. Ainsi, le jab molassie de Trinidad partage-t-il avec le mas gwosiwo mentionné plus haut la substance dont il s'enduit le corps : un mélange de gwosiwo (mélasse) et de suie. Le molasses devil trinidadien, comme son nom le suggère, se noircit lui aussi de mélasse contrairement aux jabs-jabs, diablotins en costume de satin décoré de petits miroirs et de strass qui les rapprochent des diables rouges de la Martinique où le papa djab se déplace en compagnie de ses $t i$ djab (diablotins). Leur tenue rouge est agrémentée de grelots et de petits miroirs. Le djab wouj, autre déclinaison du thème diabolique, est affublé de cornes et de miroirs et sa queue fait retentir des grelots. Les caractéristiques du jab molassie trinidadien ne portent pas tant sur les détails vestimentaires, à l'image des " mas a Congo » vêtus d'une redingote usée mais de bonne coupe, d'un short kaki ou d'un pantalon court, arborant des chapeaux melon aux bords frangés, des képis sans visière ou des bonnets rouges et serviettes de toilette nouées autour de la tête; le jab molassie exhibe fièrement ses cornes, ses chaînes et une serrure de bois munie de clefs entourant sa ceinture ; il brandit à bout de bras une fourche et au bas de son pantalon est accrochée une queue en fil métallique.

Mélasse, suie, tambour et trompette

L'évocation de la mélasse, par sa couleur foncée et par la référence à la fête, suggère une mise en parallèle avec le caouynage amérindien et la 
teinture de genipa dont s'enduisaient les participants à cette manifestation. À cette occasion, le visage se couvrait d'une poudre noire dessinant des moustaches recoquillées et des cernes autour des yeux ; les corps étaient maculés de stries à la couleur de la teinture de genipa. À une époque historique correspondant à celle des caouynages, les passagers à destination des Caraïbes, au départ de la France, se grimaient lors d'une fête ou bacchanale célébrée par les officiers du navire au moment du passage de la ligne du tropique du Cancer. Chacun s'habillait de façon grotesque et bouffonne. Les officiers du navire s'armaient de tridents, de harpons et d'autres instruments de marine, d'autres couraient aux poêles, broches, chaudrons, lèchefrites et semblables ustensiles de cuisine et se barbouillaient le visage avec le noir qu'ils prenaient au dessous des marmites. Tous sautaient de joie au son des tambours et des trompettes ${ }^{6}$.

\section{Lune cornue et mas à cornes}

Plus disséminés dans la Caraïbe sont les mas a konn, mas cornus qui formaient à Trinidad les cattle bands ou les cows bands, les Mardi-gras à cornes en Haïti ou cowhead, personnage du Roots Jonkonnu jamaïcain. Cette tradition vivace parmi les employés des abattoirs était maintenue à Trinidad par les Vénézuéliens qui se couvraient le corps de feuilles sèches de bananier et parcouraient la ville en chargeant un bullfighter. À l'image des cows bands, les mardi-gras à cornes du carnaval des Cayes en Haïti chargeaient et faisaient pousser des cris aux petits enfants. Muni d'un masque représentant entièrement la tête de l'animal, le corps du « mardigras à cornes » n'était pas recouvert de feuilles mais d'un long vêtement en tissu, contrairement aux «Fey a Bannan et Konn a Bef » des « group a po », mais il était muni d'un fouet qu'il faisait claquer pendant ses déplacements.

\section{Mokozonbi}

Le mokozonbi, comme les « mas " précédents, est largement diffusé. Dans l'iconographie guadeloupéenne des années 1950, il apparaît vêtu d'un vêtement long, une robe dissimulant les échasses sur lesquelles il est juché. Le détail marquant est le chapeau haut de forme conique surmonté d'un bâton autour duquel s'enroule un ruban. La longueur des échasses semble inférieure à 3 pouces mais a varié et a largement dépassé cette hauteur au cours des années. La relève de mokozonbi est aujourd'hui assurée en Guadeloupe et, contrairement au passé, par une majorité de filles. Le Moko Jumby trinidadien qui appartient à la tradition John Canoe se hissait

6. Cité par Corinne Landais Raboteur 
sur des échasses pouvant atteindre 10 à 15 pieds. Il portait un chapeau élaboré, rehaussé de plumes, et se faisait quelquefois accompagner par un nain dépourvu d'échasses pour accentuer le contraste.

Ces exemples sont surtout indicatifs de figures anciennes car, dans un
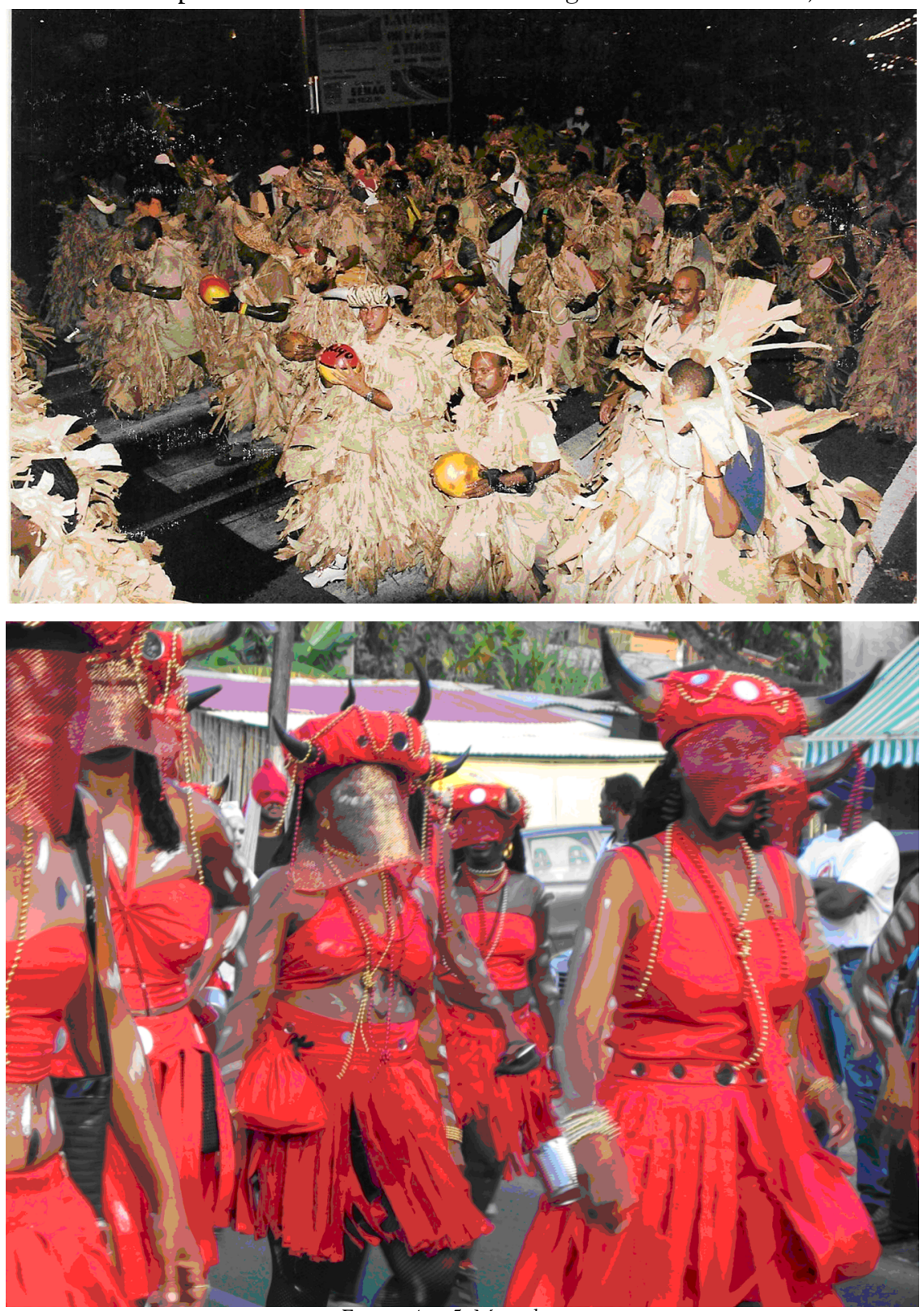

Figures 4 et 5. Mas a konn. 
registre moins éloigné mais plus ou moins perpétué et pour ne s'en tenir qu'à la proximité entre Trinidad et la Guadeloupe, s'affichaient les bébés/ baby dolls, les matelots saouls/Bad Behaviour Sailors, les «mas a ruban »/ Maypole. À cette liste, le carnaval de la Martinique ajoute, outre les diables et diablotins mentionnés plus haut, le personnage de Caroline-zié-loli, conjointe impavide ramenant au foyer un époux enivré en le portant sur son dos ; l'effet comique tient à la superposition des corps et à leur indéfinition.

\section{Contes, légendes et expression théâtrale}

La prégnance des chants particuliers au carnaval - Dja la ka mandé on ti manmay dé ti manmay (le diable réclame un enfant, deux enfants...) -, des contes et des légendes accompagnant la fête, viennent renforcer la stature des protagonistes qui survivent outre le carnaval à travers la tradition orale ; d'ailleurs, des personnages de contes, dont la diablesse, font partie des acteurs carnavalesques. Cette dernière prend l'allure d'une belle femme aguichante qui sous ses jupes dissimule un pied de bouc (sur le thème de la diablesse, voir en ligne, Kasika, chant : « la diablesse est la rue, elle monte, elle descend »). Les mariages burlesques donnent lieu à une expression théâtralisée en mettant en scène les acteurs d'une comédie basée sur la dérision. Dans le passé, le ressort comique avait trait au religieux puisque la mariée avait fauté avant le mariage mais refusait de se départir de sa couronne de fleurs d'oranger (signe de virginité). La perpétuation de la tradition burlesque privilégie aujourd'hui l'inversion de traits physiques et implicitement celle des rôles sociaux (voir, en ligne, " Mariage burlesque », groupe Point d'Interrogation). Toujours dans ce registre, chaque année des acteurs anonymes exposent à la foule des situations tragi-comiques : mari bafoué, mère en recherche de la paternité de son rejeton, situations politiques et économiques cocasses.

«L'assassin et le malheureux », forme de théâtre de rue autrefois intégré au carnaval, est une pièce chantée sans accompagnement musical ; composée de quatre strophes, elle relate les faits opposant le prince à son assaillant. Des saynètes se performaient aussi à Trinidad, des skitts interprétés pendant le carnaval ou la semaine précédente. Dame Lorine, figure marquante, se hissait sur les planches à minuit, sur le lieu de préparation des " mas », pour se mêler à une parodie de défilé élégant de la classe aristocratique du XVIII siècle. La dernière représentation eut lieu en 1955 non sans léguer à l'expression théâtrale comme ailleurs dans la Caraïbe, le sens de la dérision et du tragi-comique. 


\section{Nouvelles modalités de carnaval}

Du carnaval émergent de nouvelles modalités d'échanges et un circuit inversé de transfert entre la métropole et la périphérie. La création des carnavals de New York et de Notting Hill a marqué le coup d'envoi d'une petite industrie d'exportation de costumes au départ de Trinidad. À Paris, le carnaval n'est distant que de quelques mois du carnaval tropical dont les dix années d'existence furent célébrées en 2012 (outre le carnaval de Paris, on dénombre les carnavals de Dunkerque, de Cholet, celui des Gones et des Magnauds de la région Rhône-Alpes, d'Albi, d'Auxonne en Côte d'or, ainsi que le Carnaval vénitien d'Annecy et le Carnaval Biarnés - Béarnais - de Pau). À cette occasion, 60 groupes franciliens et des outremers ont pris part à la manifestation, dont 40 d'Île-de-France, 9 de Guadeloupe, 4 de Martinique, 3 de Guyane et 2 de la Réunion (voir, en ligne, la présentation générale du Carnaval tropical de Paris). Il s'est agi, pour les groupes issus des Antilles, de la Guyane et de l'Océan Indien, d'une participation éphémère, contrairement aux groupes franciliens résidant dans l'Hexagone mais qui, par les costumes et la musique, partagent les mêmes traditions.

En Gironde, la forte concentration d'étudiants antillais a impulsé en 2007, en marge du Carnaval des Deux Rives, le carnaval de Bordeaux, qui a acquis de l'ampleur au cours des ans (voir, en ligne, "Quatre nuits de fête à l'antillaise pour le Carnaval de Bordeaux »). Si les costumes et les thèmes traditionnels ne sont pas de rigueur mais bien souvent le produit de la cyberculture, la musique, bien que diversifiée, reste ancrée avec des artistes antillais de la scène reggae-dancehall, dépêchés sur place pour l'occasion. Outre Paris et Bordeaux, Nice accueille également des célébrations antillaises (voir, en ligne, Kasika, carnaval de Nice), tandis que, entraînés par une dynamique inverse, les touristes de la métaspora affluent dans les îles pendant cette période festive.

Aujourd'hui, dans notre monde globalisé, en proie à une logique d'improvisation de l'espace et du temps, soumis à l'accélération des migrations, des communications, des échanges, des déplacements, le tissu patrimonial se redéploie et certains pans se situent à l'intersection de la globalisation et de la métasporisation. À l'heure où la communication à distance tendrait à devancer celle en face à face, où les transferts physiques cèdent leur prépondérance par rapport à ceux virtuels, s'observe un patrimoine partagé constamment enrichi par des transferts transnationaux virtualisés dont les limites semblent imprévisibles. 


\section{Références}

Bettleheim, Judith, 1984, "The Jonkonnu Carnival : Its relation to Caribbean and African masquerades ». Jamaica Journal 10 (2-4) : 20-27. Bortolotto, Chiara (dir.), 2011, Le Patrimoine culturel immatériel. Enjeux d'une nouvelle catégorie, Paris, Éditions de la Maison des sciences de l'homme.

Cabrera, Lydia, 1989, El monte. La Havane, Editorial letras Cubanas.

Cabrera, Lydia, 1972, Cuentos negros de Cuba. Madrid et Miami, coll. del Chichereku en el exilio.

Connor, Geraldine et Max Farrar, 2004, « Carnival in Leeds and London : Making New Black British Subjectives » : 255-269. Dans Milla Cozart Riggio (dir.), Carnival : Culture in Action. The Trinidad Experience, New York, Routledge.

Coridun, Victor, 1930, Le carnaval de Saint Pierre. Fort-de France, Imp. R. Illemanu.

Cozart Riggio, Milla, 2004, Carnival : Culture in Action. The Trinidad Experience. New York, Routledge.

Cowley, John, 1996, Carnival, Canboulay and Calypso. Traditions in the Making. Cambridge, Cambridge University Press.

Crowley, Daniel, 1956, «The Traditional Masques of Carnival ». Caribbean Quarterly 4 (3-4) : 194-223.

Des Rosiers, Joël, 2013, Métaspora. Essai sur les patries intimes. Montréal, Triptyque.

Gaignebet, Claude, 1994, Le carnaval. Essais de mythologie populaire. Paris, Payot.

Green, Garth et Philip Scher, 2007, Trinidad Carnival. The Cultural Politics of a Transnational Festival. Bloomington, Indiana University Press.

Hill, Errol, 1972, The Trinidad Carnival. Mandate for a National Theatre. Austin et Londres, University of Texas Press.

Nabajoth, Eric et Michèle Montantin, 1991, Vie et mort de Vaval. Pointeà-Pitre, Association Chico-Rey.

Nurse, Keith, 2004, "Globalization in Reverse : Diaspora and the Export of Trinidad Carnival ». Dans Milla Cozart Riggio (dir.), Carnival : Culture in Action. The Trinidad Experience : 245-244. New York, Routledge.

Poulot, Dominique, 2006, Une histoire du patrimoine en Occident. XVIII - XXI ${ }^{e}$ siècles, du monument aux valeurs. Paris, Presses universitaires de France. Pradel, Lucie, 2000, African Beliefs in the New World. Trenton, NJ, Africa World Press.

Pradel, Lucie, 2013, Le patrimoine immatériel entre solidarité, transmission et communication transnationale, thèse de doctorat, Université Laval. 
Raboteur, Joël et Corinne Landais Raboteur, s.d., « Essai d'appréhension de la valeur économique d'un événement culturel majeur en Guadeloupe : le Carnaval ». Esprit critique, en ligne : http://www.esprit-critique. $\mathrm{com} /$ fiscalite/essai-dapprehension-de-la-valeur-economique-dunevenement-culturel-majeur-en-guadeloupe-le-carnaval

Roheler, Gordon, 1990, Calypso and Society in Pre-Independence Trinidad. Port of Spain, Rolher.

Smith, Laurajane, 2006, The Uses of Heritage. Londres et New York, Routledge.

Smith, Laurajane et Natsuko Akagawa (dir.), 2009, Intangible Heritage. Key Issues in Cultural Heritage. Londres et New York, Routledge.

Turgeon, Laurier, 2003, Patrimoine métissé. Contextes coloniaux et postcoloniaux. Paris et Québec, Éditions de la Maison des Sciences de l'Homme et Presses de l'Université Laval.

Turgeon, Laurier (dir.), 2009, L'esprit du lieu. Entre le patrimoine matériel et immatériel. Québec, Presses de l'Université Laval.

Voegeli, Juste-Constant, 1994, La musique dans le carnaval haïtien. Aspects urbains et ruraux. Thèse de doctorat, musicologie, Université de Montréal.

\section{Références en ligne}

Akiyo (http://www.youtube.com/watch?v=kHTryLgmk8c; http://www. youtube.com/watch?v=819_ZnRcpMc; http://www.youtube.com/ watch?v=7SiTwFXrknY)

«Arété sa »: Admiral T, Mas Moul Massif, DJ Fano (http://www.youtube. $\mathrm{com} /$ watch?v=FOhlpBgecx 4 )

Art floral, Carnaval de Guadeloupe, 2007 (http://www.dailymotion.com/ video/x19lzo_carnaval-de-guadeloupe-2007_music)

Atafaya Sainte-Anne, Abymes 2013 (http://www.youtube.com/ watch? $=$ TH6m6kdPnYo)

«Ben démaré », Rimèd razyé : féyaj pou premyé dlan ! http://www. guadeloupe.franceantilles.fr/actualite/sante/rimed-razye-feyaj-poupremye-dlan-248844.php)

« Bwa Bandé », Carnaval tropical de 2013 (http://www.youtube.com/ watch?v=vq34TghxoUo)

«Chantons les louanges », Cactus Cho (http://www.youtube.com/ watch?v=gM80kRJtkNU).

«Char pikan », Extrait 3, Carnaval tropical, Paris 2013, (http://www. youtube.com/watch?v=w4LKqD2B7vE)

«Choukaj Mas Paris » (http://www.youtube.com/watch?v=jeo2cfC04kY) 
«Concours Synthé.mpg ( (http://www.youtube.com/ watch? $=$ F2IC1KYsEKk)

Hommage à saint Georges chevalier par les artisans, Carnaval de Guadeloupe, 2007, $2^{\mathrm{e}}$ partie (http://www.dailymotion.com/video/ x19hsg_carnaval-de-guadeloupe-2007-part-2_music?start=3)

«Karukéra Stee », steel band de Guadeloupe (http://www.youtube.com/ watch?v=FO5bJPbTL1U)

Kasika, carnaval de Nice (http://www.youtube.com/watch?v=K6tVrOkHaCO)

Kasika, Mardi gras à Basse-Terre (chant : « la diablesse est la rue, elle monte, elle descend ») (http://www.youtube.com/watch?v=SqT52jnK5qM)

Kasika, «nwel a fon kako » (http://www.youtube.com/watch?v=oJhWO9LR9o)

«Mariage burlesque », groupe Point d'Interrogation (http://www. guadeloupe.franceantilles.fr/actualite/culture-et-patrimoine/ils-se-sontmaries-et-16-02-2010-67613.php).

«Mas ka kle» (http://www.youtube.com/watch?v=5dl9IenyoTE)

«Mas Mélé » (http://www.youtube.com/watch?v=-hnRundw558)

Mas Moul Massif (http://www.youtube.com/watch?v=AkcDZ3Jfe-M)

«Miyo et Maskadom » à Longjumeau (http://www.youtube.com/ watch? $=$ Xh5E3a6jkHk)

Mutuelle Mare Gaillard, historique (http://www.maregaillard.com/home. html)

«Nasyion a neg mawon », (http://www.youtube.com/ watch? $=7 \mathrm{pE} 7 \mathrm{RCOBbQU})$

«Pikan final », 2013 (http://www.youtube.com/watch?v=7RI555lWVyg)

Présentation générale du Carnaval tropical de Paris (http://www. carnavaltropicaldeparis.fr/pdf/presentation.pdf)

"Quatre nuits de fête à l'antillaise pour le Carnaval de Bordeaux » (http:// www.sudouest.fr/2012/03/01/quatre-nuits-de-fete-a-l-antillaise-pour-lecarnaval-de-bordeaux-647381-4976.php)

« Stars \& Tany's Girls », Carnaval Guadeloupe 2013, Parade du Mardi gras à Basse-Terre, (http://www.youtube.com/watch?v=nFB1aunmQUo).

«TV97.Net», Concours de Musique (http://www.youtube.com/ watch?v=cG9tw1Orufg)

Utilisation de la fibre de coco, Carnaval de Guadeloupe, 2007, 4e partie (http://www.dailymotion.com/video/x19hsk_carnaval-de-guadeloupe2007-part4_music?start=3) 